\title{
LA DISCRIMINACIÓN DE LA MUJER EN LAS ÓRDENES BUDISTAS
}

\author{
THE DISCRIMINATION OF WOMEN \\ IN BUDDHIST ORDERS
}

\author{
María Jesús Alonso SEOANE* \\ Universidade da Coruña
}

\begin{abstract}
RESUMEN: Este artículo realiza una revisión histórica sobre una parte poco estudiada del budismo como es la desigualdad de género. A través las tradiciones y normas de las distintas ramas del budismo, se trata de visibilizar el papel de las mujeres en esta religión y averiguar si esta discriminación se encuentra en la base de su filosofía o ha sido consecuencia de su evolución. A partir de las reflexiones de la obra de Rita Gross (2005), El budismo después del patriarcado, se indaga en algunos puntos controvertidos sobre el papel de las mujeres en el budismo antiguo en un intento de contribuir a esa incipiente "cantera" de estudios de lo religioso en clave feminista actualmente en auge. Desde la perspectiva metodológica de los imaginarios sociales, se analiza la discriminación de las mujeres en las órdenes budistas en tres aspectos concretos: i) la evidencia de la existencia de ordenes monacales femeninas en la época del Buda; ii) el debate sobre las reglas adicionales para el monacato femenino y iii) reflexiones sobre la discriminación femenina en el budismo y su superación.
\end{abstract}

\footnotetext{
*E-mail: chas@udc.es
} 
Palabras Clave: Historia de las religiones, budismo, feminismo, historia social, mujeres.

Авsтract: This article makes a historical review on a little studied part of the Buddhism like the inequality of gender. Through traditions and rules of the different branches of Buddhism, the paper draws attention towards the role of women in this religion and tries to find out if this discrimination is in the base of its philosophy or has been a consequence of the evolution. From the reflections of the work of Rita Gross (2005), Buddhism after Patriarchy, we will inquire into some controversial points about the role of women in ancient Buddhism in an attempt to contribute to this incipient "quarry" of studies of the religious matters in a feminist key, which is currently booming. From the methodological perspective of the social imaginary, the discrimination of women is analyzed in the Buddhists orders in three particular aspects: i) the evidence of the existence of monastic feminine orders in the times of Buddha; ii) the debate about the additional rules for feminine monastic order and iii) reflections about the feminine discrimination in Buddhism and its overcoming.

KeYwords: History of religion, Buddhism, feminism, social history, women.

\section{Introducción}

Este artículo efectuará una revisión sobre una parte poco estudiada del budismo como es la desigualdad de género. La finalidad es destacar el papel de las mujeres en un espacio del que han sido especialmente relegadas. Las religiones forman una importante base a la hora de conformar valores sociales, influyendo en la mayoría de los sistemas. La perspectiva de género puede contribuir a explicar los elementos que propiciaron la subordinación femenina en cada religión. En concreto, se analizará el papel de las mujeres en el budismo centrándose en el budismo antiguo e indagando en las diferencias existentes entre las dos vías. De manera específica, se trata de visibilizar el papel de las mujeres, averiguar si la desigualdad de género en el budismo ha sido inherente a su filosofía y principios o si se ha ido añadiendo y establecer posibles líneas a seguir en un futuro.

En España, los estudios de género y religión sobre el budismo son prácticamente inexistentes. El budismo se introduce en nuestro país como práctica 
religiosa a final del siglo XX y no sería hasta la concesión de notorio arraigo en 2007 cuando empieza a tomar auge. El Ministerio de Justicia encargó un estudio de minorías religiosas que empezó su investigación en los noventa. Si echamos un vistazo a sus contenidos solo las Universidades de Aragón y Euskadi dedican apartados a las mujeres en las minorías. El género en la religión continúa siendo poco estudiado en cuanto dejamos la "cantera" de la teología feminista sobre la religión mayoritaria en occidente: el cristianismo.

A fin de obtener información hay que acudir a literaturas con más tradición, como la anglosajona. En ese sentido, la obra de Rita Gross (2005) supone la revisión más amplia efectuada sobre budismo desde una perspectiva feminista que se haya llevado a cabo. A partir de las reflexiones extraídas de su libro $E l$ budismo después del patriarcado analizamos los puntos más controvertidos sobre el papel de las mujeres en el budismo en un intento de contribuir a esa incipiente «cantera» de estudios de lo religioso desde un enfoque en clave feminista que está comenzando a ampliarse, también en nuestro país, a otras religiones.

\section{Marco teórico}

La relación entre religión y feminismo ha sido controvertida estando más polarizada que nunca. Por una parte, el feminismo ha optado desde sus inicios por revisar textos bíblicos a los que acudían como «autoridad superior» para reivindicar el sinsentido de que la política y la sociedad mostrasen favoritismo por los varones. Las revisoras repasaron los testamentos para sustentar lo absurdo de considerar inferior a la mujer, para cuestionar el génesis mostrando la existencia de opciones más favorables a las mujeres que habían sido descartadas (Stanton, Candy y otras 1997) En la segunda ola, Simone de Beauvoir en su obra El segundo sexo (1998) acudió a la religión, entre otras disciplinas, para mostrar la falta de fundamento razonable para perjudicar al género femenino; aunque todas ellas hubiesen contribuido a reproducir el problema de la desigualdad concluyendo que «no se nace mujer, se llega serlo». La frase indica la construcción social del género; pero, como muestra, prejuicio y misoginia son mantenidos en el tiempo en cada una de las disciplinas revisadas.

En la actualidad se multiplican las opiniones sobre la imposibilidad de mezclar feminismo y religión. Por una parte, se continúa la tradición de revisar textos 
religiosos. La ubicación geográfica de las autoras propició que la mayor producción de estudios de religión con perspectiva de género se encuentre en la teología feminista cristiana, centrada en revisar textos y llevar a cabo una reconstrucción feminista de los orígenes cristianos (Schussler, 1989). Posteriormente se revisan otras religiones del libro con temas recurrentes: pecado original, prohibición de las mujeres para ejercer responsabilidades o la masculinidad de la imagen de Dios (Castilla de Cortázar, 2016) Puede decirse que «han aportado una visión femenina o lo femenino al quehacer teológico» (Consuelo Vélez, 2001). En el polo positivo del binomio entre género y religión destacamos «que el gran avance de los estudios feministas a la religión ha sido incorporar la experiencia de las mujeres a un conocimiento que se creía neutro» (Tarducci, 2001).

Otro enfoque percibe el binomio como inútil o limitante, subrayando el papel de legitimación de la desigualdad que tradicionalmente mantienen las religiones. Un ejemplo sería «From the pulpit to the Street», centrado en las manifestaciones antiabortistas de grupos ultracatólicos (Cornejo y Pichardo, 2017).

\section{La escasez de estudios de budismo y género en España}

Con anterioridad, algunas ramas católicas habían estudiado el budismo, siendo los jesuitas los primeros en tener contacto en el siglo XVI. Uno de los primeros estudiosos en España es el profesor López- Gay a quien debemos la literatura de los años setenta, que incluye interesantes capítulos sobre las mujeres que serán comentados aquí. Llegó a vivir en Asia dedicando años a la investigación budista. Mucho tiempo ha tenido que pasar para contar con investigaciones españolas sobre el tema. La falta de apertura en la Dictadura hace que no se estudiase más religión que la católica. En cuanto a las demás, pocas áreas traspasaban la frontera entre Oriente y el Occidente español. Una de las primeras doctorandas en traspasar esa frontera fue María Teresa Román, pionera en la investigación y divulgación del budismo con una tesis en 1994 sobre el Prajnaparamita. A partir de ahí le siguen Abraham Vélez con una tesis sobre la filosofía madhyamika (2001), publicada como libro en (2003). María Jesús Alonso (2008), que analizó medios de comunicación para ver el imaginario social sobre el budismo a partir de análisis de contenidos. Francisco Díez de Velasco (2013), quien radiografió la 
situación del Budismo en España. Pero no es hasta ahora cuando aparecen estudios españoles sobre budismo y género. Es importante destacar la aportación de Ana Iborra, (2014), quien defendió un TFM de estudios feministas buscando en el budismo una clave para superar el debate entre Sheila Benhabib y Judith Butler sobre el sujeto y la agencia. Lejos de quedarse en una revisión filosófica, de por sí interesante, empleó esta reflexión como búsqueda de un budismo utilizable a la teoría feminista (debate entre modernidad y posmodernidad) y a cuestiones de identidad sexual. Concluye que el budismo a través de su filosofía (rama vajrayana) y su práctica puede ser útil a la teoría feminista. (Iborra, 2014). Una perspectiva complementaria considera a «las ciencias religiosas un campo favorable para que la perspectiva feminista presente sus contribuciones» (Pádua, 2018) buscando "comprender en qué medida los abordajes feministas pueden colaborar con el estudio de las religiones» (Pádua, 2018).

\section{Metodología}

Siguiendo a Berger y Luckman (1966), entendemos que la realidad se construye socialmente. Siendo así, las religiones influirían en la sociedad, pero también son un producto de ella. Estos autores afirman que la sociología del conocimiento debe analizar los procesos de construcción social. Pero no es posible abarcar la totalidad de una realidad desde el interior del sistema, para ello es necesario un observador de segundo orden (Luhmann, N., 2007), así que nos posicionaremos en ese lugar de observación apoyándonos, por una parte, en la hermenéutica y por otra, en la teoría de los imaginarios sociales.

La neutralidad científica, la dimensión objetiva, cede su sitio a las subjetividades y particularidades, más o menos organizadas. "A fin de cuentas, se trata de estudiar textos y contextos que afectan a la realidad de la fe y a las creencias [...] esto, como puede adivinarse, encierra tantos riesgos como riesgos encierra la supuesta neutralidad científica. De nuevo, se impone la necesidad de una seria hermenéutica de la sospecha» (Navarro, 2010).

La hermenéutica de la sospecha es necesaria, pero no suficiente. Ha mostrado capacidad de deconstrucción, pero no garantiza la re-construcción necesaria 
para resultar útil. Por ello, es de interés enfocar el estudio desde la perspectiva de los imaginarios sociales, donde al tiempo que se cuestionan los lugares desde los cuales se observa, también se toma conciencia de su proceso de construcción, percibiéndola como algo dinámico. El profesor Pintos explica que «los imaginarios sociales serían aquellas representaciones colectivas que rigen los sistemas de identificación y de integración social y que hacen visible la invisibilidad social» (Pintos, J.L, 1995). Apuntar hacia las evidencias invisibles es completamente necesario cuando se trata de indagar en los discursos sobre un fenómeno social, preguntarnos por su sentido e interacciones, su articulación en los diferentes procesos comunicativos y el ámbito de producción de esos discursos y significados. En el estudio del imaginario social en torno a la discriminación de las mujeres en las órdenes budistas, nos basaremos en tres aspectos concretos, enfocando tanto lo que damos por seguro como indicios con respecto a: i) la evidencia de la existencia de ordenes monacales femeninas en la época del Buda; ii) el debate sobre las reglas adicionales para el monacato femenino y iii) reflexiones sobre la discriminación femenina en el budismo y su superación.

\section{El budismo temprano: De la ordenación de bikkunnis ${ }^{1}$ al cisma}

\subsection{Los hechos}

Se ha recogido que el Buda se negó a ordenar mujeres en su primera petición cuando Mahapajapati Gotami pidió la entrada en la orden junto a 600 mujeres. También está probado que hubo orden femenina en un tiempo y sociedad (la india de hace 2.500 años) en que las mujeres vivían para sus familias, dependían de sus maridos y a veces llegaban a inmolarse con ellos tras su muerte. Las mujeres no tenían posibilidad de escapar de sus destinos, justificados en una concepción pre-budista del karma que explicaba su situación en el momento presente ni podían abandonar su casa para estudiar.

Conocemos la existencia de órdenes femeninas por las escrituras del Canon Pali, en las cuales no solo se hace referencia a ellas, sino que se recogen poemas

\footnotetext{
1 Monjas.
} 
(therigata) que describen sus experiencias liberatorias. Estos textos, escritos en primera persona, quedaron recogidos en las escrituras antiguas. Para el budismo theravada, o de los antiguos, las escrituras en pali son las únicas que pueden considerarse como enseñanzas del Buda. La literatura de las therigatas, recopilada en este Canon, parece dejar claro que las mujeres alcanzaban espacios de igualdad en lo referente a experiencias místicas. Experiencias que son la mayor meta del individuo en una sociedad budista, ya que alcanzar la iluminación supone una trascendencia del espíritu y la mente humanas a niveles de sabiduría y altruismo superiores a cualquier meta. Por este motivo, la visibilización de las mujeres en el espacio religioso es un tema interesante en esta religión, al suponer un reconocimiento explícito de igualdad real. Los logros quedaron patentes en estos poemas:

¡Soy libre! Estoy completamente liberada

De las tres cosas torcidas:

De la argamasa, pestilencia

Y de las torceduras del viejo esposo.

Estoy liberada de nacimiento y muerte,

Todo lo que renueva las existencias,

Ha sido desarraigado.

(Thig 1,11 Muttatherigatha - Versos de la Venerable Mutta)

Punna, está llena de las cosas sublimes,

Como la Luna [está llena] el día quince del mes,

Con la completa sabiduría, derrumbó totalmente,

La masa de la oscuridad [de la ignorancia].

(Thig 1,2 Muttatherigatha - Versos de la Venerable Mutta) 
Los poemas documentan que ellas conseguían alcanzar el nirvana ${ }^{2}$. Recientemente, las therigata han sido traducidas por completo a nuestro idioma en un libro con el mismo título.

La disciplina es una de las virtudes que ayudan al aspirante al nirvana a conseguir su propósito de liberarse de la ignorancia, motivo por el cual fue recopilado un código de disciplina monástica denominado vinaya $a^{3}$. De algunas normas recogidas en él pueden realizarse interesantes lecturas, pues el vinaya nos permite deducir que la inclusión femenina en la sangha produjo cierto malestar en la sociedad de su tiempo. Un ejemplo serían las normas adicionales para las monjas.

Algunos estudiosos occidentales han visto discriminación en las normas. Mas estas teorías están siendo desbancadas por budologas procedentes de universidades asiáticas. Sus interpretaciones apuntan a que cada norma debería ser interpretada en su adecuado contexto si pretendemos no caer en las generalizaciones y el prejuicio.

\subsection{Mujeres en el budismo temprano}

Tal como sugieren algunas profesoras asiáticas, no podemos explicar una sociedad de hace 2.500 años con nuestros patrones mentales modernos y occidentales. Es importante situar las cosas en su marco histórico y cultural para ver el significado que tenían en esa época. La mayor parte de argumentos a favor de la existencia de un «budismo patriarcal» surgen de las reglas adicionales para monjas. Pero no tienen por qué ser interpretadas como discriminatorias. Una

2 Liberación absoluta del sufrimiento conseguida a través de la práctica de las virtudes y la meditación. En todas las formas de budismo, alcanzar el nirvana es el comienzo de la santidad o bien la santidad absoluta (según tradiciones) y tiene un enorme reconocimiento social al ser estas personas semejantes al Buda y superiores al plano de los dioses. Posteriormente, ha sido cuestionada en varios países la mayor dificultad de las mujeres para alcanzar el nirvana. Algunos mantienen actualmente la convicción de que es más fácil para los hombres, motivo por el que las mujeres aspiran a un renacimiento como hombre para estar más próximas a esa meta.

3 Código de disciplina monástica. Forma parte del Canon Pali.

4 Comunidad monástica, que habitualmente vive en monasterios y ocasionalmente en retiros en la naturaleza. 
opción apuntada es que el Buda establecía continuamente excepciones a las normas a fin de adecuarlas a las circunstancias y sociedad.

La Dra ${ }^{a}$. Kabilsingh, profesora de filosofía y religión en la universidad de Thammasat, Bangkok, mantiene la teoría de que muchas normas iban siendo añadidas a medida que se planteaba la necesidad de defender la igualdad de las mujeres dentro de la shanga ${ }^{5}$. Ella sostiene que muchos de los bikkhus ${ }^{6}$ de la época estaban acostumbrados a ver a las mujeres con un determinado rol social que pretendían mantener en las comunidades monásticas. Esperaban que, al igual que ocurría fuera, las mujeres limpiasen y planchasen las ropas de los monjes. Estas cuestiones motivaban que las reglas fuesen adaptándose, pero el Buda afirmó, desde el principio, la igualdad de las mujeres en cuanto a su capacidad. Así consta entre los argumentos que le dio a su discípulo, Ananda, sobre la negativa del maestro a admitir mujeres en la primera petición. A su pregunta directa: ¿es que no tienen las mujeres el mismo potencial de iluminación que los hombres, no pueden alcanzar el nirvana?, el Buda responde que sí tienen el mismo potencial de iluminación y pueden alcanzar el nirvana. Esta afirmación abría un camino de igualdad social y el reconocimiento explícito del potencial de sabiduría que suponía el nirvana. No hay que olvidar que esa experiencia no eleva al ser humano a la altura de los dioses, sino que los supera. La cosmología budista mantiene la creencia en seis planos de existencia, siendo el de la budeidad superior al de las deidades.

Tras la admisión femenina, algunas de las normas suplementarias para las mujeres pretendían una igualdad real. La profesora ve en este tipo de normas una forma de protección contra la desigualdad social. Para la autora, las normas vendrían a suponer algo similar a lo que hoy llamaríamos una discriminación positiva.

La académica tailandesa explica con otros ejemplos las excepciones para mujeres. Con fines equitativos se estableció otra norma que impedía a las mujeres compartir sus donativos con los monjes varones. Esta norma, dice, se introdujo tras llegar al Buda la información del desmayo de una monja anciana que donaba su comida (recibida mediante limosna) a un joven monje vago que no salía a mendigar la propia. Cuando el carruaje de un noble pasó al lado de la anciana esta cayó al suelo a causa de la debilidad. Entonces el hombre se quejó ante el

5 Comunidad monástica.

6 Monjes varones. 
Buda de que permitía a una anciana salir a mendigar y además lo compartiese con alguien que podía ocuparse de su sustento. Al conocer ese suceso, el Buda dictó una norma que impedía a los bikkus tomar las limosnas de las monjas. La norma fue interpretada desde Occidente como si ellas fuesen percibidas como "casta inferior» de la cual un monje no admitía limosna. Cualquier conocedor del budismo sabe que el Buda se opuso a la sociedad de castas de su época. Esto invalidaría ese tipo de interpretación, aunque la sociedad de castas existía en ese momento. Así, explica la profesora, cada una de las normas surge de una necesidad concreta que no es posible interpretar en términos de desigualdad por parte del fundador.

Rita Gross, por su parte, discrepa con que las normas adicionales fuesen añadidas, algo con lo que no concuerdo. En primer lugar, porque no da explicaciones que motiven su afirmación. En segundo, porque otros que han tenido acceso directo al estudio de los originales opinan lo contrario de modo razonado. Una de las reglas especialmente controvertidas es la Patimoskha ${ }^{7}$ que se encuentra en el capítulo 23 del vinaya y que obliga a declarar públicamente las faltas cometidas. Según esta regla, una monja no puede obligar a un monje a declarar públicamente sus faltas y cuando así se haga se considerará incorrecto; en cambio, cuando un monje pida lo mismo con respecto a una monja, esta deberá hacerlo; y pedirlo así se considerará correcto.

De esta norma puede inferirse que surgió un conflicto entre las órdenes femeninas y masculinas. Existen varios indicios para considerarlo así, ya que se recogen enseñanzas en las que los monjes pedían consejo al Buda sobre cómo evitar la tentación del deseo con respecto a las mujeres. Las referencias negativas a las mujeres aparecen también en textos del sabio Shantideva. Según el Dalai Lama, las referencias obedecen a esas consultas sobre cómo evitar el deseo. Explica que tales afirmaciones no deben ser tomadas de modo literal. Aunque nos parezca extrańo, visualizar algo en negativo es un método usado en el budismo. Se hace, por ejemplo, para abandonar el apego por el propio cuerpo con objeto de trascenderlo algún día. Todas las ramas del budismo creen en la reencarnación, siendo su principal motivo el apego al propio cuerpo. Los tibetanos practican meditaciones con visualización donde se imaginan a sí mismos como un amasijo de carne y huesos con los que no hay que identificarse. Este método también es relatado por Gross (2005) para explicar que, en el budismo, ver algo como negativo no significa despreciarlo, sino trabajar con el apego hacia ese objeto o

Código o reglas. 
persona. Para el budismo, consciencia e identificación son conceptos diferentes, como bien explica Iborra (2014).

Pensar que la integración femenina en la sangha fue sencilla estaría lejos del sentido común, ya que la sociedad, y el monacato formaba parte de ella, no estaba dispuesta a asumir sin fisuras un cambio en roles de género mantenidos durante siglos. Por otra parte, la mujer era una pieza esencial en su familia, cumplía funciones de producción y reproducción y, en la sociedad de castas, tenía una subordinación al marido.

Por otra parte, distintas teorías mantienen que las normas para las mujeres fueron añadidas, así lo considera el profesor López-Gay (1974), quien declaró con contundencia:

Buda impuso a las primeras monjas las «ocho reglas fundamentales». Algunas de ellas suponen un largo tiempo de experiencia. Sin duda fueron redactadas posteriormente, sistematizadas y atribuidas a Buda: $1^{\text {a }}$, el respeto a los monjes; [ $2^{\mathrm{a}}$ etc] $7^{\mathrm{a}}$, bajo ningún pretexto debe abusar o injuriar a un monje, $8^{a}$, no se permite una admonición de una monja a un monje, sí al contrario. Quizás el grupo más antiguo de estas reglas fundamentales lo formen aquellas que miran al trato con los monjes. Sobre la octava volvió repetidamente Buda, prohibiendo la admonición de las monjas a los monjes (López-Gay, 1969).

El propósito de la vida monástica es reunir las condiciones óptimas para el crecimiento espiritual. Una posible interpretación a la reticencia de algunos monjes a admitir mujeres podría radicar en el papel tan importante otorgado a la renuncia y a los placeres sensuales. No renunciar a ellos era una prueba evidente de no dominar las emociones y tener una mente liberada. La obligación de convivir con mujeres (inicialmente, las shangas eran mixtas) supondría a algunos monjes un permanente recuerdo de las debilidades que querían superar. Esta explicación hace razonable la existencia de normas que especifican que un monje no puede estar a solas en determinados lugares con una mujer u otras indicaciones que van en la dirección de evitar tentaciones. Posteriormente las sanghas se separaron.

El profesor Harvey (2000) relata que poco después del fallecimiento del Buda (400 a. C), se celebró una «recitación comunal», un concilio de arahats en Rajagaha, para poder ponerse de acuerdo sobre el contenido del dharma y 
del vinaya que el Buda había legado en tanto que maestro. Unos sesenta ańos después del primero, se celebró un segundo concilio en Vesali con el propósito de reprender a ciertos monjes con un comportamiento laxo en relación a diez puntos. Dieciséis años después se produjo el primer cisma de la shanga que, hasta entonces, se había mantenido unida. Esto sentaría el inicio de la división entre las dos vías principales que perviven hasta el momento: theravada y mahayana. Pese a que el budismo es considerado una religión mística, si se le aplicase la metodología de Palmisano (2014) para clasificar religiones podría considerarse que tiene dos vertientes, una más mística que otra. De confirmar el encaje podríamos argumentar que todas las religiones han tenido una actitud más integradora con la mujer en sus vertientes místicas. En este caso, la más favorable sería la mahayana.

Las investigaciones recientes indican que la causa más probable del cisma fue un intento de ampliar ligeramente el número de reglas monásticas. La preocupación por el rigor y la unidad monástica pudo haber inducido a una de las secciones del shanga a incorporar algunas reglas nuevas en la selección del vinaya que se refería a la conducta, la forma de vestir y el comportamiento en público de los monjes (Harvey, 2000).

Parece claro que existió malestar en una parte de la sangha de bikkhus y no solo por ese tipo de normas. Mantenemos esto porque así lo han interpretado también varios autores. Eliot apunta a este malestar de los primeros tiempos, a pesar de lo cual:

Parece que el monacato femenino estuvo muy floreciente durante la vida de Buda. En parte se debía al entusiasmo que despertaba la personalidad de Buda y la de algunos de sus primeros discípulos, como Ananda. A raíz del primer concilio budista, donde Ananda fue acusado de haber abierto la puerta del monasterio a las monjas, pasó una crisis. En los primeros siglos aún mantuvo una cierta vitalidad (Eliot, 1962).

Según los expertos, las controvertidas reglas pudieron ser perfectamente introducidas en una etapa posterior. Lo que sabemos con seguridad es que el Buda no dejó nada escrito y sus enseńanzas se fueron recogiendo en las reuniones de esos primeros concilios. El Canon Pali es el texto más primitivo, por tanto, la afirmación de López- Gay cabría como explicación a la norma de la «admonición de monjas a los monjes», dado que es coherente tanto con la visión histórica como con el sentido general de las enseñanzas. Además, ofrece argumentos razonables en cuanto a que estructura y estilo de esas reglas no siguen el mismo 
patrón del resto. En la misma obra del profesor aparecen varias referencias más a la orden de bikkhunnis que destacan el importante papel de las mujeres, su igualdad en cuanto a dominio del dharma, así continúa explicando:

Se conservan, con todo, dos casos de monjas que gozaban del carisma de ser voz de Buda (buddhahvacana) y podían instruir a toda clase de personas. Dhammadïnna era una monja que respondía siempre con profunda filosofía a los que le preguntaban; cuando el Buda se enteró, hizo propia la doctrina de la monja ${ }^{8}$. El otro caso de monja buddhavacana es una religiosa de Kanjagalâ a la que visitaban muchos preguntando la interpretación de verdades difíciles99 (López-Gay, 1974).

Sería erróneo concluir que las monjas, al menos al principio, hayan estado relegadas al segundo plano. Parece que, inicialmente, tuvieron las mismas oportunidades y que demostraban aprovecharlas enseñando temas difíciles. Los datos históricos no dejan lugar a duda. Asunto diferente es que los logros femeninos no hayan trascendido a las biografías o que la creación de normas de convivencia monacal haya ido adaptándose a las sociedades a las que llegaba, del mismo modo que ocurrió con los métodos de enseñanza. Concuerdo con Gross en que la doctrina budista debió adaptarse continuamente a patrones patriarcales.

\subsection{Evolución de la sangha}

Cada tradición fue adaptando las normas del vinaya a los países por los que el budismo se iba extendiendo, dando lugar a los cánones tibetano, chino, etc. Lejos de reducirse, las reglas adicionales para bikkunis se multiplicaron en todos los cánones, pasando de las 8 adicionales a sumar 50 ó 70 . Parece que la tendencia a la subordinación femenina era un hecho en cada país. Las normas parecen obedecer a adaptaciones sociales.

Luego, dentro de la corriente theravada, las monjas budistas nunca llegaron a adquirir carta de ciudadanía. En Ceilán, aunque el monacato femenino fue introducido por la hermana de Manida, que ordenó a las primeras monjas, no

8 MN disc, 44 Cf todo el cant. 12 del Therîgâhâ, donde se explica el don que tenía de "la perfecta penetración analítica de las formas y del significado de la doctrina".

9 AN 10,3,27; en las reglas de las monjas (Pâcittiya no 49) se les prohíbe estudiar y enseñar materias mundanas.

ÉNDOXA: Series Filosóficas, n. 42, 2018, pp. 137 -157. UNED, Madrid 
logró echar raíces ${ }^{10}$. El mismo fenómeno se puede notar en Birmania o en Laos, (donde la orden de bikunnis no ha existido jamás). En los países de tradición mahayana, el monacato femenino ha conocido, sobre todo al comienzo, cierto esplendor, que poco a poco se ha ido oscureciendo. La China del siglo IV ofrece las primeras noticias sobre el movimiento monástico, reglas y grandes figuras de monjas. En el año 343, Ho- Ch'ung fundó el primer monasterio de monjas budistas; su sobrina, la emperatriz Ho, levantó otros monasterios; la importancia de las monjas a finales del siglo IV se desprende de las medidas que tuvo que tomar la corte imperial para controlarlas: «significa el comienzo de la influencia de las monjas en la corte y en el Gobierno, un influjo que a comienzos del siglo $\mathrm{V}$ adquirió proporciones alarmantes» ${ }^{11}{ }^{12}$ (López-Gay, 1974).

Como se aprecia en el texto, las mujeres con más dificultad a la hora de ser aceptadas en las órdenes se ubican en los países del theravada. Atendiendo a las diferencias metodológicas entre ambas vías no es difícil comprender el alcance de las perturbaciones que las mujeres parecían ocasionar a la bikkhu sangha. Molestias que dependían del modo en que eran percibidas por los monjes, como fuente de distracción o deseo. En la metodología theravada se enfrenta una perturbación evitándola. En el mahayana, evitar un problema no supone traspasarlo, debiendo enfrentarlo. La metodología pudo contribuir a que las mujeres hayan tenido menos dificultades, e incluso una época de esplendor, durante la extensión del budismo hacia donde se extendió el mahayana. Esto podría llevarnos a pensar en una mejor posición para las mujeres en esta vía. No obstante, siguiendo el desarrollo del monacato femenino se aprecian indicios de que el mahayana también contó con una fuerte discriminación, evidenciada en hechos como la multiplicación de normas adicionales en los países del norte. El escaso número de ordenaciones completas de mujeres y a que tengan un techo de cristal en la mayoría de los países. Pero, sobre todo, a la creencia en una mayor facilidad de alcanzar nirvana desde un cuerpo de varón.

Posteriormente, con la creciente divergencia dentro del mundo budista, fueron prevaleciendo las opiniones y discursos contrarios a las mujeres. La orden de mujeres no pudo abolirse por completo pero sus logros sufrieron restricciones. Llegó a decirse que los Budas siempre encarnan como varones, que todos los puestos importantes de la jerarquía y de la mitología solo

\footnotetext{
RAULA, WAPOLA. The History of Buddhism in Ceylon. Colombo 1956.

ZÜRCHER, E. The Buddhism Conquest of China.

LÓPEZ - GAY, J. Op.Cit.
} 
podrían ser ocupados por hombres, e incluso que las mujeres no podían llegar a la iluminación. Esas duras doctrinas probablemente no fueron características del budismo originario, pero circularon ampliamente en el momento en que el budismo empezó a escindirse (Gross, 2005).

El paso por un cuerpo de hombre para poder alcanzar el nirvana supone una postura supremacista, pues la pretensión de estar más próximos a la iluminación presentaría a los hombres como superiores.

En el mahayana, incluyendo su derivación vajrayana, se admite la idoneidad para enseñar como maestro al margen del monacato. Así, muchas escuelas tibetanas cuentan con sabias y maestras (Allione, 1991) que no han sido ordenadas. La capacidad de un maestro no depende de su ordenación monástica para tres de las cuatro escuelas tibetanas. No obstante, las mujeres han sido menos visibilizadas y se conocen pocos personajes históricos maestras de varones con mayor reconocimiento. Yeshe Tsogyal, Machig Lapdron, Jomo Memo, Machig Ongjo, Drenchen Rema o A-yu Khadro son algunos ejemplos de biografías recuperadas de sabias tibetanas.

El tibetano es una modalidad particular de budismo. Continuó otorgando un lugar relevante a lo femenino no como maestras o monjas, cuyo número es muy inferior al de varones y menos destacado; sino en cuanto parte necesaria de la unidad como una suma de principios complementarios, algo tomado probablemente del panteón hinduista, aunque también existía en el taoísmo. Brahman o lo absoluto en India o el Tao en China, son conceptos de unidad que para ser alcanzados precisan trascender la dualidad aparente. Siendo así, podemos encontrar figuras de deidades femeninas que no existen en el budismo theravada. Tara es la deidad femenina más popular y reverenciada del budismo tibetano, formando parte de las cinco budas femeninas ${ }^{13}$. Otras escuelas del mahayana, como el zen, no tienen la particularidad de representar parejas que simbolizan los principios de una mente iluminada, al no provenir del budismo indio, sino del chino. No obstante, mantienen una reverencia

13 Una de las diferencias entre las dos vías es que el Mahayana considera budas a todos aquellos que han alcanzado la iluminación; admitiendo, por tanto, numerosos budas femeninos y masculinos. Para la tradición antigua, el término Buda se reserva para Sidharta Gautama. Según esta tradición, quien alcanza el nirvana es un arahat (bendito o santo), alguien que está en el camino, pero no habría alcanzado una budeidad completa. El budismo theravada admite que todos los seres puedan tener experiencias de nirvana que los elevan a un estado de sabiduría, pero no admite el panteón de budas que pueden llegar a darse en el mahayana. 
hacia lo femenino en la recitación de sutras como el de la Prajna Paramita, o en figuras como Kuan Ying, que incluye el principio de la energía femenina (ying) en su propio nombre, siendo representada casi siempre como mujer. Podría decirse que el mahayana ha mantenido de algún modo lo femenino, aunque sea más en lo simbólico que en lo real.

\section{Momento presente}

Pero ¿qué ha pasado con las mujeres de a pie? ¿Con sus posibilidades de aprender, de enseñar o ser ordenadas en igualdad con sus compañeros? En Japón, las puertas del monacato se abrieron a las mujeres en el S. XII gracias al maestro Dohën. Pero el número de maestras de zen es muy inferior al de varones, calculándose en torno a un $10 \%$ del total de la sangha.

En cuanto a las tibetanas, el Dalai Lama ha reiterado su compromiso de apoyo a las mujeres creando mejores universidades donde alcanzar la totalidad de votos. Hay que decir que el monacato tibetano de monjas ha tenido más posibilidades fuera que dentro de Asia, siendo una de las primeras ordenaciones completas la de Pema Chödron, en 1981.

Uno de los principales escollos que han tenido que salvar las mujeres para progresar en el camino de la ordenación ha sido la particular obligación de requerir doble ordenación: por un grupo de monjas de su linaje, y otro de monjes de la misma orden. Con frecuencia esto ha sido imposible debido a que pocos países han mantenido los linajes femeninos a lo largo del tiempo. En ocasiones desaparecieron y en algún caso no llegaron a existir.

Debido a la concepción budista de que la realidad no es más que un proceso en movimiento continuo, el papel de las mujeres ha evolucionado ambas vías. Muchas mujeres se han movido para retomar el compromiso y el ejemplo de las enseñanzas del Buda con la igualdad y muchos hombres han dado apoyo en ese sentido. En primer lugar, en Taiwán, donde se restableció la ordenación femenina completa. Los casos son diferentes en cada lugar, a veces para retomar la ordenación bikkuni ha tenido que acudirse a monjas de otros países para reimplantar la tradición. En otras ocasiones se ha aceptado la ordenación de bikkunis solo por bikkus, sin participación de mujeres de la misma tradición cuando ello era imposible debido a la desaparición previa de la orden femenina. 
Birmania intentó restablecer la orden de mujeres en 1930 y en 1970, ambas con nulo resultado; el gobierno no intentó facilitar la recuperación de la femenina hasta los ańos 80 .

El budismo de tradición tailandesa está recorriendo el mismo camino que otras modalidades. La primera ordenación femenina y la orden de bukkhuni se ha restablecido fuera del país en 2009, concretamente en Australia. Algunos de los más puristas niegan su validez por no haber podido contar con la parte de ordenación femenina del linaje. No obstante, la tradición tailandesa ha encontrado apoyo en una parte de su sangha masculina. Desde siempre han existido hombres que apoyaron la entrada de mujeres, como Ananda o Dohen. Bikkhu Sujato, monje de la tradición tailandesa del bosque, ha escrito numerosos artículos explicando la pertinencia de apoyar la orden femenina. Incluso tradujo textos del pali que sustentan la idea de que una ordenación de bikkunis efectuada por bikkus es perfectamente válida y aceptada por el vinaya. Explica que en la sangha antigua lo normal era ordenar a las bukkhunis; primeramente, por parte femenina, y en segundo lugar por la parte masculina que tenía que aceptarlo. Esta es la norma, pero «a veces ese proceso podría ser interrumpido, por ejemplo, si había peligros que impidiesen viajar al bikkhu sangha para la ordenación» (Sujato, 2009) y continua: "Durante ese intervalo ella sería aceptada de un lado». Finaliza explicando que:

[...] sin embargo, sigue siendo un hecho indiscutible que la concesión para la ordenación por sólo los bikkhus existe y nunca se anuló. Esto contrasta con la situación en el proceso a la ordenación de bikkhu. Pero la ordenación de bikkhunis es menos definitiva. La concesión para la aceptación sólo de bikkhus está claramente establecida y nunca se revocó, pero el texto continúa como si hubiese dejado de aplicarse. Yo entendería esto mayormente como un ligero descuido del tratamiento de las bikkhunis. No se puede discutir que tal ordenación sólo por los bikkhus no sería la mejor práctica, según el vinaya pali. Pero tampoco se podría mantener que no estuviese permitido (Sujato, 2009).

Puede afirmarse que las mujeres están recuperando los espacios que les habían sido negados. A raíz de una conferencia internacional de mujeres en Bodgaya en 1987 bajo el patrocinio del Dalai Lama, se formó la Asociación internacional Sakyadhita, que reúne a monjas de todas las tradiciones. La organización ha celebrado congresos femeninos y ya llevan XVI. La Asociación cuenta con sedes en diez países, entre ellos España, y dispone de publicaciones en varias lenguas. 
En su web aparece un apartado dedicado a deidades femeninas de varias tradiciones y personajes femeninos relevantes del budismo. Asimismo, disponen de otro apartado para iniciativas femeninas y se informa sobre los distintos países del mundo que van reintegrando las órdenes de bukkhuni.

\section{Conclusión}

Las mujeres fueron admitidas en la orden de la primera etapa alcanzando los logros más valorados y enseñando sobre ello. Poner el foco en que las causas de la exclusión no tuvieron base filosófica o religiosa es importante a la hora de restructurar el imaginario femenino. La importancia de mostrar estos hechos obedece a dos motivos:

Porque la historia de la humanidad hubiese sido diferente de haber continuado dando a las mujeres en protagonismo social que tuvieron en otras épocas.

Porque, en el caso del budismo, la implicación va más lejos del simple reconocimiento social, dado que la capacidad de alcanzar el nirvana eleva a los humanos sobre las deidades. Ello implica un reconocimiento de los valores humanos universales sobre las distintas categorizaciones por género o cualquier elaboración social. No cabe mayor evidencia del papel que el budismo suponía para la población femenina, que el elevado número de mujeres que lo siguieron (600 pidieron la primera entrada en la orden del Buda) y en la China del siglo IV las monjas comenzaban a tener influencia política, considerándose su influjo alarmante a principios del siglo V. Otros indicadores en la misma dirección son los poemas donde las propias mujeres dejaron constancia de sus logros. Del mismo modo, apreciamos evidencias bibliográficas sobre cómo la historia ha ido recogiendo los logros de sabios y excluyendo los de sus maestras. Siguiendo la evolución del monacato femenino, vemos que cada forma de budismo tuvo su propia manifestación de discriminación, en el theravada invisibilidad y exclusión, en el mahayana degradación del potencial femenino.

Se propone seguir la trayectoria histórica en diversos países y tradiciones, así como comparativas posteriores con otras religiones a fin de comprender las causas y estrategias en cada forma específica de desigualdad. Los diversos países budistas evolucionaron en base a selecciones de lo que consideraron valioso: la opción de auto-liberación, descartando las enseñanzas de igualdad y de la trascendencia de opuestos. Pero el foco está puesto ahora en la recuperación de la 
esencia, contribuyendo así a generar futuras realidades centradas en la inclusión. El papel de las budistas antiguas y modernas, orientales y occidentales, y su determinación de crear su futuro es innegable. Las mujeres budistas, conocedoras de la inexistencia de una realidad que funcione por sí misma, están recuperando protagonismo. Han podido hacer esto al no limitarse a una de-construcción de partes pre-seleccionadas o filtradas, sino centrándose en la re-construcción de la realidad que quieren ver en el futuro.

\section{Bibliografía}

Aguado, J. (2016). Therigata. Barcelona: Ed. Kairós.

Allione, T. (1991). Mujeres de sabiduría. Barcelona: Ed. La liebre de marzo.

Alonso, M. J. (2008). Budismo y medios de comunicación. Santiago de Compostela. USC.

3/4 (20I0) «Los géneros imaginarios de lo sagrado. El budismo en el marketing publicitario» Madrid. Aposta Revista de Ciencias Sociales número 46.

Beauvoir, S. (1998). El segundo sexo. Valencia: Cátedra.

Berger, P y Luckmann, T. (1966). La construcción social de la realidad. Madrid: Amorrortu.

Cady Stanton, E. (ed.) (1997). La Biblia de la mujer. Valencia: Ediciones Cátedra.

Castilla de Cortazar, B. (2016). «Mujer y Teología: la cuestión de la imagen de Dios». Madrid. ARBOR Ciencia, Pensamiento y Cultura. Vol. 192-778, marzo abril 2016, a302.

CoRnejo, M y PIChardo, I. (2017). «From the pulpit to the street: ultraconservative religious position against Gender in Spain». En Anti-gender campaings in Europe (Kuhar and Paternotte ed.) Rowam and Littlefield International.

Consuelo Vélez, C. (2001). «Teología de la mujer, feminismo y género». Río de Janeiro, Brasil: Theologica Xaveriana 140.

Coomaraswarny, A. K. (1994.) Buddha y el evangelio del budismo. Barcelona: Ed. Paidós Orientalia.

Déz de Velasco, F. (2013). El Budismo en España. Madríd. Akal.

Einei Döhen (1990). Puntos esenciales en la práctica de la vía. (Gakudo Yojinshu). Madrid: Miraguano ediciones. 
Euıot, Сн. (1962). "Hinduism and Buddhism», Vol. I. En BEAL, S. Comparative Arrangement of two Translation of the Buddhist Ritual for the Priesthood, known as the Pratimoksha or Patimokkhan, Londres: Pali. Journal of Assam Reserch Society 13.

Gross, R. (2005). El budismo después del patriarcado. Madrid: Ed. Trotta.

Harvey, P. (2000). El budismo. Cambridge: Cambridge University Press.

Iborra Asencio, A.M. (2014). «La Dakini, un camino hacia el empoderamiento femenino». Valencia: Universidad de Valencia.

Jones, Сн. (1999). Buddhism in Taiwan: Religion and the State 1660-1990. Honolulu: University of Hawaii Press.

Kabilsinghl, CH. «Historia de la Orden de bikkhunis». En http://bosquetheravada. org

Lottermoser, F. (1991). «Monjas budistas en Tailandia». Sakyadhita Newsletter, Vol.2, no.2. http://www.sakyadhita.org

López - GAY, J. (1974). El monacato budista. Madrid: Ed. Católica. Serie Semina verbi, sobre religiones no cristianas.

3/4 (1969). «Pâtimokkha o 'Reglas' del monacato budista según fuentes de la literatura Pâli», Boletin de la Asociación Española de Orientalistas, vol. V. (pp. 113- 137).

Luhmann, N. (2007). Introducción a la teoría de sistemas. México: Ed. Universidad Iberoamericana.

Navarro, M. (2010). «Biblia, mujeres y feminismo. II Parte: El nuevo testamento en el cristianismo primitivo", Ilu Revista de Ciencia de las Religiones. Madrid.

3/4 (2007). «Teología y feminismo. Pensamiento y autoridad», http://www.mujeresenred.net. «Teología y feminismo. Pensamiento y autoridad».

Maioli, E. (2011). «La religión como objeto de estudio sociológico. Una revisión de la teoría sociológica de Emile Durkheim, Max Weber y Niklas Luhman sobre la religión». Buenos Aires: IX jornadas de Sociología.

Menzan Zuinó (1990.) La clara luz del ser (Jijuyu Zanmai). Madrid: Miraguano Ediciones.

Pádua Freire, A. E. (2018). "Perspectivas de Género nos estudos da Religao: contribuÇoes das ciencias feministas». Dosiè: Estudos de Religao e Epistemologias. Vol V, número 13 Belo Horizonte, Brasil.

Palmisano, S. y Jonveaux (2014). «Sociology of Monasticism: Between Innovation and Tradition», Brill, Leiden- Boston. Annual Review of the Sociology of Religion, vol. 5

Pintos de Cea- Naharro, J.L. (1995). «Orden social e imaginarios sociales (una propuesta de investigación)». Barcelona: Papers revista de Sociología. Vol 45. 
Raula Wapola (1956). The History of Buddhism in Ceylon. Colombo: MD Gunasena.

Roman López, M.T. (2014). Análisis del problema de la conciencia en la Prajnaparamita Hridaya Sutra. Madrid. UNED (Tesis doctoral no publicada).

Sanz Hernández, A. (2014). «La Religión- Género como mediación de socialidad y visibilidad. Un estudio de caso de las mujeres pertenecientes a comunidades religiosas en Aragón». Madrid, Migraciones núm. 35.

Sanz Hernández, A. (2009). «Mujer y Religión». En Construyendo Redes. Minorías religiosas en Aragón (coord. Gómez Bahaillo). Madrid: Icaria editorial.

Schussler Fiorenza, E (1989). En Memoria de ella. Una construcción feminista de los origenes del cristianismo. Bilbao: Desclée de Brouwer.

Sujato (2009). «Ordenación de monjas por monjes», en https://sujato.wordpress.com

TARDUCCI, M. (2001). «Estudios feministas de Religión: una mirada muy patriarcal», Sao Paulo, Cadernos Pagu núm. 16.

Enviado: $10 / 04 / 2018$

Recibido: $16 / 11 / 2018$

Este trabajo se encuentra bajo una licencia de Creative Commons ReconocimientoNoComercial-SinObraDerivada 4.0

\section{(1) 9 \\ (2) $\mathrm{HD}$}


